\title{
Differential effects of post-weaning juvenile stress on the behaviour of C57BL/6 mice in adolescence and adulthood
}

Journal Article

Author(s):

Peleg-Raibstein, Daria; Feldon, Joram

Publication date:

2011-03

Permanent link:

https://doi.org/10.3929/ethz-b-000099767

Rights / license:

In Copyright - Non-Commercial Use Permitted

Originally published in:

Psychopharmacology 214(1), https://doi.org/10.1007/s00213-010-1991-8 


\title{
Differential effects of post-weaning juvenile stress on the behaviour of $\mathrm{C} 57 \mathrm{BL} / 6$ mice in adolescence and adulthood
}

\author{
Daria Peleg-Raibstein • Joram Feldon
}

Received: 30 April 2010 /Accepted: 4 August 2010 /Published online: 28 August 2010

(C) Springer-Verlag 2010

\begin{abstract}
Rationale There is evidence that events early in postweaning life influence brain development and subsequent adult behaviour and therefore play an important role in the causation of certain psychiatric disorders in later life. Exposing rodents to stressors during the juvenile period has been suggested as a model of induced predisposition for these disorders.

Objective This is the first study to examine behavioural and pharmacological changes in adolescence and adulthood following juvenile stress in mice.

Materials and methods Two cohorts of mice were simultaneously exposed to a stress protocol during postnatal days (PND) 25-30. Behavioural assessments reflecting emotional functions, cognitive functions, and psychostimulant sensitivity were then carried out at two time points: one cohort was tested during adolescence (PND 39-54; adolescent group), and the second cohort was tested during adulthood (PND 81-138; adult group).

Results In the adolescent mice, juvenile stress significantly attenuated conditioned freezing and led to decreased anxiety-like behaviour in the elevated plus maze, whereas no effect was observed on these tests in the adult mice. In contrast, adult mice exhibited poor avoidance learning following juvenile stress. When tested during adulthood, the mice stressed during the juvenile period showed a sensitised response to amphetamine compared to controls, whereas the response during adolescence was similar in stressed and control animals.
\end{abstract}

D. Peleg-Raibstein $(\bowtie) \cdot$ J. Feldon

Laboratory of Behavioural Neurobiology,

Federal Institute of Technology Zurich,

Schorenstrasse 16,

CH 8603 Schwerzenbach, Switzerland

e-mail: daria.peleg@behav.biol.ethz.ch
Conclusions Our results suggest that exposure to stressors during the juvenile period can exert long-term effects on the brain and behaviour and that these effects differ depending on whether the animals are tested during adolescence or adulthood.

Keywords Early life · Juvenile · Stress · Mice · Behaviour · Amphetamine

\section{Introduction}

In humans, epidemiological studies have indicated that stress experienced during early life is associated with an increase in the prevalence of psychopathologies such as depression, post-traumatic stress disorder, and anxiety disorders (Heim and Nemeroff 2001; Luecken and Lemery 2004; Weiss et al. 1999) later in life (Heim et al. 2004; Kessler and Magee 1993; Penza et al. 2003). This relationship is complex, and the underlying neurobiological mechanisms that mediate the contribution of stressful experiences to the manifestations of mental illness are not well understood. Human studies are limited in their ability to examine the specificity of the relationships between childhood adversity and adult psychopathology as different forms of maltreatment often co-occur in the same individual. Moreover, most human studies are retrospective and correlational and therefore prevent the clear establishment of causal relationships between specific childhood experiences and adult psychopathology. Furthermore, because of ethical considerations and limitations associated with human experimental studies, animal models are highly valuable in providing appropriate control and evaluation conditions to study particular aspects of the above-mentioned disorders. If an animal model is associated with symptomatology similar 
to that observed in humans, it can provide further understanding of the neurobiological development and mechanisms of the disease.

In both mice and rats, there have been various attempts to model stress early in life. While there is considerable literature regarding pre- and early postnatal manipulations, only a limited number of studies have been performed during the post-weaning phase encompassing the developmental juvenile period. One general classification system of adolescence in rodents encompasses three stages: the early adolescence period of postnatal days (PND) 21-34, the mid-adolescence period of PND 34-46, and the late adolescence period of PND 46-59 (Tirelli et al. 2003). Another commonly used system considers PND 28-46 the adolescence period (Spear 2000).

Recently, different laboratories have studied the impact of stress during adolescence upon adult behaviour in rats. However, the variety of early stress paradigms, the exact time period of stress application, and the duration of the exposure to stress all differ significantly in the literature. Therefore, it is difficult to obtain a consistent view of longterm behavioural outcomes from these studies [for review (Hall 1998)]. Furthermore, some of these studies tested animals immediately after the stress protocol (Kabbaj et al. 2002; Mathews et al. 2008), while others tested the animals during the adult period (Avital et al. 2006; Avital and Richter-Levin 2005; Tsoory et al. 2007; Tsoory and Richter-Levin 2006). Taking all of these factors into consideration makes it difficult to create a coherent view that would enable us to understand the possible underlying neurobiological mechanisms. For example, early life stress in rats has been shown to impair spatial learning in the water maze (Avital et al. 2006; Avital and Richter-Levin 2005; Isgor et al. 2004; Tsoory et al. 2007), enhance startle reactivity (Avital et al. 2006; Avital and Richter-Levin 2005; Bazak et al. 2009; Cohen et al. 2007; Maslova et al. 2002; Tsoory et al. 2007), reduce avoidance responses (Tsoory et al. 2007; Tsoory and Richter-Levin 2006), reduce exploratory behaviour in a novel environment (Avital et al. 2006; Avital and Richter-Levin 2005; Tsoory et al. 2007; Tsoory and Richter-Levin 2006), and increase anxiety levels in the elevated plus maze (Avital et al. 2006; Bazak et al. 2009; Cohen et al. 2007; Tsoory et al. 2007). There have also been reports, however, showing no differences in exploratory behaviour (Toledo-Rodriguez and Sandi 2007; Toth et al. 2008) or anxiety levels (Pohl et al. 2007; Toledo-Rodriguez and Sandi 2007). Juvenile stress has not been found to affect cognitive function in adulthood (Toth et al. 2008).

These studies have shown that stress in early life results in long-term altered stress responses in the brain and behaviour and may have an impact on psychological functioning later in life. As indicated above, most work has been performed in rats. However, considering the extensive use and availability of transgenic mouse models for genetic components of psychiatric disorders, we were interested in determining behavioural changes after juvenile stress in mice. The goal of the present study, therefore, was to evaluate the impact of exposure to a series of aversive stressors during the juvenile period (PND 25-29) in male C57BL/6 mice on specific behavioural stress-related neuropathologies during adolescence (PND 39-54) and adulthood (PND 81-138). We used a stress protocol during the juvenile period composed of 5 consecutive days of exposure to different stressors such as forced swimming, a platform shaker, water deprivation, restraint stress, and exposure to a rat. Thereafter, we evaluated the effects of the juvenile stress on emotional behaviour and cognitive function during adolescence and adulthood using the elevated plus maze, the open field, fear conditioning, two-way active avoidance, the Morris water maze, and locomotor activity in response to a systemic injection of amphetamine.

\section{Materials and methods}

\section{Subjects}

Inbred male C57BL $/ 6$ mice (stressed (STRESS), $n=14$; control $(\mathrm{CON}), n=14)$ were obtained from our in-house, specificpathogen-free breeding colony derived from C57BL/6N breeding pairs from Charles River Laboratories (Germany).

Four days before beginning the stress procedure, 21-dayold male mice were weaned and individually caged in Makrolon type II cages $(267 \times 207 \times 140 \mathrm{~mm}$-floor area 370) (Tecniplast, Milan, Italy). The mice were kept under controlled conditions $\left(21^{\circ} \mathrm{C}\right.$ at $55 \%$ relative humidity) with a reversed 12-h light/dark cycle (lights on at 7:00 PM), and all tests were conducted during the dark phase. Mice were maintained with food and water ad libitum unless stated otherwise. At the age of 25 days, mice assigned to the STRESS group underwent a daily stress procedure for 5 days. After a break of 9 days (at 39 days old), seven STRESS and seven CON animals (adolescent group) began the behavioural experiments, while the others remained in their home cages until they reached adulthood (adult group). All manipulations described here were previously approved by the Cantonal Veterinary Office of Zurich and conformed to the ethical standards required by the Swiss Act and Ordinance on Animal Protection and the European Council Directive 86/609/EEC.

Postnatal stress procedure

During the juvenile period from day 25 to 29 , we carried out a stress protocol comprising 5 days of tandem exposure 
to different stressors. For all of the animals assigned to the STRESS group, the stress procedure, which varied in duration as a function of the particular stressor, started every morning at $10 \mathrm{am}$, except for the rat exposure stressor, which was carried out during the light cycle. The five different stressors were as follows:

Day 1: Forced swim: An opaque, circular water tank (diameter, $32 \mathrm{~cm}$; height, $43 \mathrm{~cm}$; water depth, $26 \mathrm{~cm})$ was filled with cold water $\left(4 \pm 1^{\circ} \mathrm{C}\right)$. The mice were carefully lowered into the water, where they had to swim for a maximum of $4 \mathrm{~min}$. If a mouse started to sink, it was removed from the water, ending the stress exposure. Following the swim stress, animals were dried and placed in a holding cage before placed back into their home cage.

Day 2: Platform shaker: The mice were placed in a box $(16 \times 10 \times 24 \mathrm{~cm})$ fixed to a shaking platform (KS 250 basic; IKA Labortechnik, Germany). Shaker stress was applied (250 cycles/min) with $1 \mathrm{~cm} \pm$ $1 \mathrm{~mm}$ amplitude of displacement per oscillation for $12 \mathrm{~min}$, after which the mice were returned to their home cages.

Day 3: Water deprivation: The mice were deprived of water in their home cages for $23 \mathrm{~h}$.

Day 4: Restraint stress: The mice were placed inside plastic tubes (internal diameter, $26 \mathrm{~mm}$ ) for $2 \mathrm{~h}$ during the dark phase of the light cycle and kept in a dark experimental room.

Day 5: Exposure to a rat: Mice were individually introduced into small cages $(22 \times 14 \times 12 \mathrm{~cm})$ that were placed into a rat home cage $(57 \times 38 \times$ $18 \mathrm{~cm})$, where the mice were exposed to a rat (male Wistar rat) for $15 \mathrm{~h}(5: 00 \mathrm{PM}$ - 8:00 AM). Holes in the plastic cages assured olfactory exposure to the predator. Water and food were available ad libitum. The mice were then housed in their home cages in the animal room for the rest of the day (8:00 AM - 5:00 PM).

Behavioural testing

Behavioural testing commenced when the mice reached either 39 days old (adolescent group) or 81 days old (adult group).

Elevated plus maze

Apparatus The elevated plus maze consisted of two exposed and two enclosed arms joined to a central square platform. Its construction has previously been described in detail (Yee et al. 2004). The maze was located in a dimly lit experimental room. The light level in the open arms of the two mazes was balanced at $30 \mathrm{~lx}$ and in the closed arms $4 \mathrm{~lx}$. A digital camera was mounted above the maze, and images captured at a rate of $5 \mathrm{~Hz}$ were transmitted to a PC running the Ethovision tracking system (Noldus Technology, The Netherlands).

Procedure The mouse was gently placed in the central square facing one of the open arms. It was then allowed to move undisturbed for $5 \mathrm{~min}$. Before each trial, the arms were cleaned with water and dried. Two anxiety-related measures were calculated: percentage of time in the open arms (time in the open arms/time in all arms $\times 100 \%$ ) and percentage of entries into the open arms (open arms entries/ entries into the open or enclosed arms $\times 100 \%$ ). In addition, the total distance travelled over the entire surface (arms and central platform) of the maze provided a measure of general locomotor activity.

\section{Open field}

Apparatus The apparatus consisted of four identical openfield arenas constructed from wood laminated with plastic with a waterproof, white finish. The arenas $(40 \times 40 \mathrm{~cm}$ in surface area) were each surrounded on all sides by a $30-\mathrm{cm}$ high wall and located in a testing room with diffused, dim lighting $(30 \mathrm{~lx})$. A digital camera was mounted directly above the apparatus, capturing images from all four arenas at a rate of $5 \mathrm{~Hz}$. The images were then transmitted to a PC running the Ethovision tracking system (Noldus Technology, The Netherlands), and locomotor activity was indexed by the cumulative displacement of the centre of gravity of the subject's surface area over successive frames, expressed in 10-min bins.

Procedure The mice were tested in groups of four. They were gently placed in the centre of the appropriate arena and allowed to explore undisturbed for $60 \mathrm{~min}$. Afterwards, they were returned to their home cages, and the arenas were cleaned with water and dried prior to testing of the next group. Locomotor activity was indexed by the distance travelled in consecutive 10-min bins. In addition, the exploration of a central region $(13 \times 13 \mathrm{~cm})$ of the open-field arena was recorded; this anxietyrelated measure may be considered an index of phobia towards open spaces (Prut and Belzung 2003).

Pavlovian conditioned freezing

Apparatus The apparatus consisted of two distinct sets of four test chambers, providing two contexts. The first set of 
chambers (context A) comprised four operant chambers (Model E10-10, Coulbourn Instruments, Allentown, Pennsylvania, USA), each installed in a ventilated, sound-insulated chest. Each chamber was equipped with a grid floor made of stainless steel rods (4 $\mathrm{mm}$ in diameter) spaced at an interval of $10 \mathrm{~mm}$ centre-to-centre. A scrambled electric shock $1 \mathrm{~s}$ in duration and $0.3 \mathrm{~mA}$ in intensity could be delivered through the grid floor (Model E13-14, Coulbourn Instruments). The chamber had a total floor area of $30 \times 25 \mathrm{~cm}$ and a height of $29 \mathrm{~cm}$, but the mouse was confined to a rectangular region $(17.5 \times 13 \mathrm{~cm})$ in the centre, defined by a clear Plexiglas enclosure. Illumination inside the chamber was provided by a house light $(2.8 \mathrm{~W})$ positioned on the panel wall $(2.5 \mathrm{~lx}), 21 \mathrm{~cm}$ above the grid floor. The second set of chambers (context B) comprised four cylindrical $(19 \mathrm{~cm}$ in diameter), Plexiglas enclosures fixed on a white plastic floor, with each located in a ventilated, sound-insulated wooden cabinet. Illumination was provided by an infrared light source instead of visible light.

Each of the eight chambers was equipped with a Sonalert (Model SC628; Mallory, USA) and a digital camera mounted $30 \mathrm{~cm}$ directly above the centre of the area of interest. The Sonalert delivered a continuous tone $(2.9 \mathrm{kHz})$ stimulus at an intensity of $86 \mathrm{~dB}$. The output of the camera was fed to a multiplexer (Model YSQ-430; Sony, Tokyo, Japan) before being transmitted to a computer running the NIH Image software (version 1.61) for real-time analysis. The algorithm of the freezing response detection procedure has been validated and fully described previously (Richmond et al. 1998). Briefly, successive digitised images $(192 \times 144=$ 27,648 pixels, on an 8-bit grey scale) obtained at a rate of $1 \mathrm{~Hz}$ were compared after appropriate thresholding. The number of pixels differing between adjacent frames was then computed. If the difference was less than $0.05 \%$ of the total number of pixels in a frame, the mouse was considered freezing in that 1 -s interval.

Procedures The protocol consisted of three distinct phases: (a) conditioning, (b) test of conditioned context freezing, and (c) test of conditioned tone freezing across days. On testing day 1 , all mice were given three conditioning trials in Context A. Each trial consisted of a 30-s tone stimulus followed immediately by a $1-\mathrm{s}, 0.3-\mathrm{mA}$ foot shock. The first trial was administered 3 min after the mice were placed into the chambers. Successive trials were administered every $3 \mathrm{~min}$. The conditioning session concluded with a final 3-min interval. On testing day 2, the mice were returned to Context A. They were placed in the test chamber for a period of $8 \mathrm{~min}$. On testing days 3 to 5 , conditioned-stimulus (CS) freezing to the tone stimulus was assessed in Context B. The tone stimulus was administered 3 min after the mice were placed into the test chamber. The tone remained on for a period of $8 \mathrm{~min}$.
Two-way active avoidance learning

Apparatus The apparatus consisted of four identical twoway shuttle boxes (Model H10-11M-SC, Coulbourn Instruments, Allentown, Pennsylvania, USA). The internal dimensions of each box were $35.5 \times 18 \times 32.5 \mathrm{~cm}$. Each box was separated into two identical compartments by an aluminium wall with an interconnected opening $(6.5 \times$ $8 \mathrm{~cm}$ ), allowing the mouse to move freely from one compartment to the other (i.e., a shuttle response). The grid floor was made of stainless steel rods (diameter, $0.4 \mathrm{~cm}$; spaced $0.7 \mathrm{~cm}$ apart) and was connected to a constant-current shock generator (Model H10-1M-XX-SF, Coulbourn Instruments). Through the grids, electric shocks of $0.3 \mathrm{~mA}$ could be delivered. The CS was an $85-\mathrm{dB}$ tone produced by a $2.9-\mathrm{kHz}$ tone module (Model E12-02, Coulbourn Instruments) placed behind the shuttle box on the floor of the shell. The shuttle response was detected by a series of photocells (Model H20-95X, Coulbourn Instruments) mounted on the side of both shuttle compartments.

Procedure The conditioning phase lasted for 1 day. Each mouse was placed in the shuttle box and underwent 100 avoidance trials administered at variable intertrial intervals with a mean of $45 \mathrm{~s}$ (range $=25-55 \mathrm{~s})$. A trial began with the onset of the noise (the CS). If the mouse shuttled within $5 \mathrm{~s}$ of CS onset, the CS was terminated, and the mouse avoided the electric shock on that trial. Avoidance failure led immediately to an electric foot shock presented in coincidence with the CS. This could last for a maximum of $2 \mathrm{~s}$, but could also be terminated by a shuttle response during this period (i.e., an escape response). The number of avoidance responses in successive blocks of 20 trials was taken as an index of avoidance learning and was submitted to statistical analysis.

Spatial reference memory task in the water maze

Apparatus The water maze consisted of a white, circular fibreglass tank $102 \mathrm{~cm}$ in diameter and $36 \mathrm{~cm}$ deep. It was filled with a fresh mixture of hot and cold tap water on each test day to a depth of $19 \mathrm{~cm}$ and a temperature of $24 \pm 1^{\circ} \mathrm{C}$. A transparent Plexiglas cylinder (diameter, $7 \mathrm{~cm}$; height, $18.5 \mathrm{~cm}$ ) was used as the escape platform, with its surface submerged $0.5 \mathrm{~cm}$ below the water surface, making it invisible to the mice. Its location, however, could be flagged by a white circular disk cue (diameter, $12 \mathrm{~cm}$ ) mounted $12 \mathrm{~cm}$ directly above the platform. A digital camera was installed above the water maze, capturing and transmitting images at a rate of $5 \mathrm{~Hz}$ to a $\mathrm{PC}$ running the Ethovision tracking system (Version 3.1, Noldus Technology, The Netherlands) to compute the escape latency and 
distance travelled on each trial, along with additional dependent measures on probe tests (see below). The water maze was positioned in the centre of the testing rooms, which were enriched with unique distal spatial cues under dim lighting conditions (12 lx as measured from the maze centre).

Procedure On days 1 and 2, the animals were tested in the visually cued task across four consecutive trials with a 1-min intertrial interval. The platform surface was $0.5 \mathrm{~cm}$ above the water level, and its presence was made visible by mounting a white disk of the same size $(7 \mathrm{~cm}$ in diameter) $12 \mathrm{~cm}$ directly above it. This cued task served to familiarise the mice with the apparatus and swimming in the water maze. It further served to screen for any nonspecific sensorimotor impairment. The platform was positioned in different locations across the four trials: in the centre of the pool and in the centre of the three quadrants not used to locate the hidden platform in the subsequent reference memory task. The starting point for releasing the mouse in the pool was constant across trials within a day but changed from day 1 to 2 . The platform locations and starting positions were counterbalanced as much as possible with respect to all betweensubjects factors. A trial ended when the animals escaped onto the platform or when $60 \mathrm{~s}$ had elapsed, at which point the animal was guided to the platform by the experimenter. The animal was allowed to spend $30 \mathrm{~s}$ on the platform. Afterwards, it was placed into a waiting cage for a further $30 \mathrm{~s}$ prior to the beginning of the next trial.

On days 3 to 5 , the animals were trained to locate the escape platform, which was now hidden under the water surface and remained in a constant location (in the middle of one of the quadrants, $22 \mathrm{~cm}$ away from the maze wall). Across the four trials in a day, the starting positions varied among $\mathrm{N}, \mathrm{E}, \mathrm{S}$, and $\mathrm{W}$ in a pseudorandom sequence. Otherwise, the testing procedures were identical to those described above. On each trial, the latency and distance swum to reach the platform (visible or hidden) were recorded.

On day 6, a 60-s probe test was conducted. In the probe test, the platform was removed from the water maze, and the animal was released into the quadrant opposite the one in which the platform had been previously located (i.e., the "target" quadrant). Performance on a probe test was evaluated by calculating the percentage of time spent and distance swum in the target quadrant on its own as well as in comparison to the other three quadrants. Measurement of annular crossings was used to evaluate more closely the accuracy of the spatial search. Annular crossings were defined as the number of crossings into the area where the hidden platform was located during training.

Reference memory in the water maze was evaluated only in adult mice as the time window during the adolescent period did not permit testing.
Locomotor response to systemic amphetamine in the open field

Apparatus The apparatus consisted of four identical openfield arenas constructed from plastic, laminated wood with a waterproof white finish $(40 \times 40 \mathrm{~cm}$ in surface area), as described above. A digital camera mounted above the open fields captured images at a rate of $5 \mathrm{~Hz}$ and transmitted them to a personal computer running the Ethovision tracking system (Noldus Technology), which calculated a mobility score defined as the distance travelled per bin in successive 15 -min bins.

Procedure First, the mice were allowed to explore the open-field arena for $30 \mathrm{~min}$ (baseline). The mice were then injected with vehicle solution (isotonic $0.9 \% \mathrm{NaCl}$ solution) and immediately placed in the apparatus. Locomotor activity was measured for $30 \mathrm{~min}$ as described above. The mice were then removed from the apparatus and were administered D-amphetamine sulphate (AMPH, $2.5 \mathrm{mg} / \mathrm{kg}$, intraperitoneally). They were immediately returned to the same arena, and the locomotor response to the acute drug challenge was monitored for a period of $120 \mathrm{~min}$. All solutions for injection were freshly prepared on the day of testing and were administered in a volume of $5 \mathrm{ml} / \mathrm{kg}$ body weight. Amphetamine (AMPH, obtained from Sigma-Aldrich, Switzerland) was dissolved in sterile $0.9 \% \mathrm{NaCl}$ saline to achieve the required dose of $2.5 \mathrm{mg} / \mathrm{kg}$.

\section{Statistical analysis}

All data were analysed using parametric analysis of variance (ANOVA), followed by Fisher's LSD post-hoc comparisons whenever appropriate. All statistical analyses were carried out using the statistical software StatView (version 5.0) on a PC running the Windows XP operating system. To better conform to the homogeneity and normality assumptions of parametric ANOVA, a natural logarithmic data transformation was performed whenever necessary. A two-tailed criterion of $P<0.05$ was used as the cut-off for statistical significance.

\section{Results}

Elevated plus maze

Adolescent mice

A $2 \times 5$ (group $\times$ bin) ANOVA of the percentage of time spent in the open arms revealed significant effects based on 
group $(F(1,12)=7.09 ; P<0.03)$ and bin $(F(4,48)=7.71 ; P<$ $0.0001)$. These effects showed that juvenile stress led to an overall increase in the percentage of time spent in the open arms and that there was a general decline in the percentage of time spent in the open arms over the 5 -min test period $(F(1,12)=$ 6.79; $P<0.03$; Fig. 1a).

A $2 \times 5$ (group $\times$ bin) ANOVA of the total distance moved in the plus maze yielded significant effects based on group $(F$ $(1,12)=5.56 ; P<0.04)$ and bin $(F(4,48)=3.42 ; P<0.02)$. These effects reflect the reduced locomotor activity in STRESS mice compared to CON mice. Moreover, the mice demonstrated habituation over bins as the session progressed (Fig. 1b).
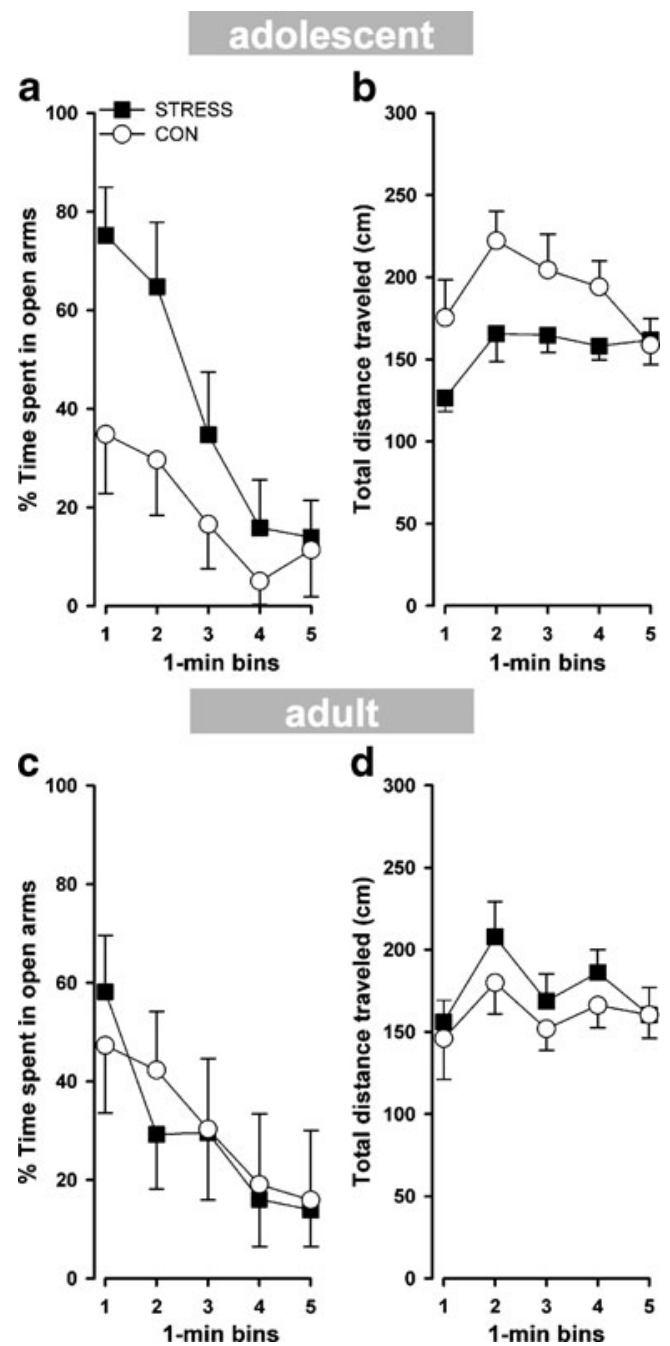

adult

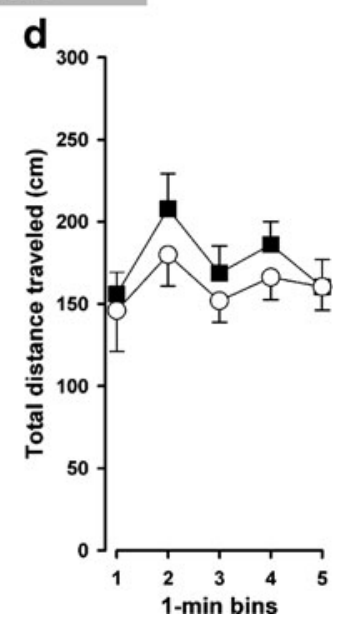

Fig. 1 Anxiety-related behaviour in the elevated plus maze. Adolescent STRESS mice spent significantly more time in the open arms than CON mice (a). In contrast, adolescent STRESS mice moved significantly less in the maze compared to the CON mice (b). Among the adult mice, CON and STRESS mice spent the same amount of time in the open arms (c) and moved the same distance in the maze (d). Error bars refer to $\pm \mathrm{SEM} ; n=7$ per group; (empty circle $)=\mathrm{CON}$; (filled square) $=$ STRESS
Adult mice

A $2 \times 5$ (group $\times$ bin) ANOVA of the percentage of time spent in the open arms yielded only a significant effect based on bin $(F(4,48)=6.60 ; P<0.004)$, reflecting a gradual decrease in the percentage of time spent in the open arms across time in both groups. We did not observe significant effects based on group and interactions between groups and bins $(F<1$; Fig. 1c).

A $2 \times 5$ (group $\times$ bin) ANOVA of the total distance moved in the maze yielded an effect based on bin $(F(4,48)=3.06$; $P<0.03$ ), indicating an increase and a decrease in activity between bins in both STRESS and CON mice (Fig. 1d). No significant effects based on group or interactions between groups and bins were observed $(F<1)$.

Open field

\section{Adolescent mice}

A $2 \times 6$ (group $\times$ bin) ANOVA of the total distance moved revealed a significant effect based on bin $(F(5,60)=32.62$; $P<0.0001$ ), reflecting the fact that the distance travelled in the arena decreased as a function of bins. The STRESS mice showed higher locomotor activity compared to the CON mice. This observation is supported by a significant effect based on group $(F(1,12)=11.31 ; P<0.006$; Fig. 2a). The total distance moved in the centre zone of the open field did not differ significantly between the groups (data not shown).

\section{Adult mice}

A $2 \times 6$ (group $\times$ bin) ANOVA of the total distance moved revealed a significant effect based on bin $(F(5,60)=27.18$; $P<0.0001)$, reflecting the fact that the distance travelled decreased as a function of bins. No significant effects based on group or interactions between groups and bins were observed $(F<1$; Fig. $2 b)$. The total distance moved in the centre zone of the open field did not significantly differ among the groups (data not shown).

Pavlovian conditioned freezing

\section{Adolescent mice}

Day 1: Tone-shock conditioning

A $2 \times 3$ (group $\times$ trial) ANOVA of the natural logarithmictransformed (ln-transformed) percentage of time spent freezing yielded significant effects based on group ( $F$ $(1,12)=7.41 ; P<0.02)$ and trial $(F(2,24)=46.75 ; P<$ $0.0001)$. These findings show that the freezing response to 


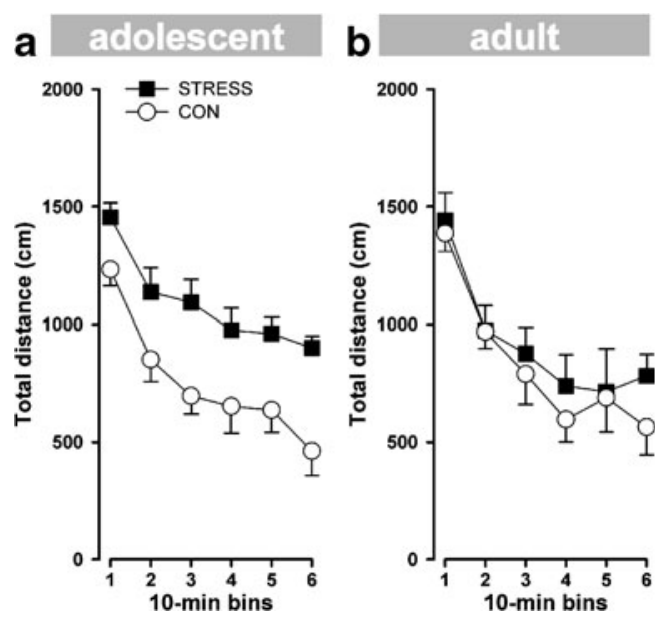

Fig. 2 Assessment of locomotor activity in the open field. (a) Adolescent STRESS mice exhibited higher locomotor activity levels in the open field compared to the CON mice. (b) Adult STRESS and CON mice did not differ in locomotor activity during the entire $60 \mathrm{~min}$ of the open-field test. Error bars refer to \pm SEM; $n=7$ per group; $($ empty circle $)=\mathrm{CON}$; (filled square $)=\mathrm{STRESS}$

the CS tone increased across the three successive toneshock pairings and that the STRESS mice exhibited a lower level of freezing (Fig. 3a).

Day 2: Conditioned freezing to context

The conditioned freezing response to context was examined across the 8 -min test period. For analysis purposes, an average of 2 -min bins was taken. A $2 \times 4$ (group $\times$ bin) ANOVA of the ln-transformed percentage of time spent freezing to context revealed a significant effect based on group $(F(1,12)=5.29 ; P<0.05)$, with the STRESS mice exhibiting lower freezing levels compared to the CON mice. No significant effects based on bin or interactions between groups and bins were detected (Fig. 3b).

Days 3-5: Expression of conditioned freezing to the tone

The conditioned freezing response to the CS tone was examined across the 8 -min test period. For analysis purposes, an average of 2-min bins was taken. A $2 \times 3 \times 4$ (group $\times$ day $\times$ bin) ANOVA of the ln-transformed percentage of time spent freezing per bin yielded significant effects based on day $(F(2,24)=10.07 ; P<0.0008)$ and bin $(F(3,36)=9.54$; $P<0.0001)$ and a significant interaction between days and bins $(F(6,72)=3.22 ; P<0.008)$. These findings indicate that conditioned freezing to the CS tone decreased within a test session and across test days. Furthermore, the analysis revealed a significant effect based on group $(F(1,12)=8.33$; $P<0.02)$, a group $\times$ day interaction $(F(2,24)=3.42 ; P<0.05)$ and a significant group $\times$ day $\times$ bin interaction $(F(6,72)=3.74$; $P<0.003)$. These effects reflect reduced freezing to the tone in the STRESS animals, which was most pronounced on day 3 (first test day) and within days at the early stage of the test
(Fig. 3c). In summary, the STRESS mice showed a clear reduction in conditioned freezing to a discrete CS.

\section{Adult mice}

Day 1: Tone-shock conditioning

A $2 \times 3$ (group $\times$ trial) ANOVA of the $\ln$-transformed percentage of time spent freezing yielded a significant effect based on trial $(F(2,24)=46.95 ; P<0.0001)$, indicating that the freezing response to the CS tone increased across the three successive tone-shock pairings (Fig. 3d). This effect was comparable between the two groups. No other effect or interaction reached statistical significance.

Day 2: Conditioned freezing to context

The conditioned freezing response to context was examined across the 8-min test period. For analysis purposes, an average of 2 -min bins was taken. A $2 \times 4$ (group $\times$ bin) ANOVA of the ln-transformed percentage of time spent freezing to context revealed only a significant effect based on bin $(F(3,36)=4.49 ; P<0.009)$. The freezing response to context increased over time, and the freezing levels were comparable between the STRESS and CON mice (Fig. 3e). No other main effect or interaction reached statistical significance.

Days 3-5: Expression of conditioned freezing to the tone

The conditioned freezing response to the CS tone was examined across the 8 -min test period. For analysis purposes, an average of 2-min bins was taken. A $2 \times 3 \times 4$ (group $\times$ day $\times$ bin) ANOVA of the percentage of time spent freezing per bin yielded significant effects based on day $(F(2,24)=17.27 ; P<0.0001)$ and bin $(F(3,36)=15.14$; $P<0.0001$ ), reflecting extinction of freezing both within and between days. The freezing levels decreased across days and within the test session. There were no statistically significant outcomes involving the different groups (Fig. 3f).

Two-way active avoidance learning

\section{Adolescent mice}

A $2 \times 5$ (group $\times 20$ min trial block) ANOVA of avoidance crosses yielded a main effect based on block $(F(4,48)=$ 23.88; $P<0.0001)$, indicating avoidance learning in both groups as a function of blocks (Fig. 4a). No other main effect or interaction attained statistical significance.

\section{Adult mice}

A $2 \times 5$ (group $\times 20$ min trial block) ANOVA of avoidance crosses yielded a main effect based on block $(F(4,48)=$ 
Fig. 3 Pavlovian conditioning to a CS tone. (a) Freezing to the CS (30 s in duration) is shown in the adolescent STRESS mice, which exhibited significantly lower levels of freezing than the $\mathrm{CON}$ mice. (b) Contextual conditioning: adolescent STRESS mice showed a reduction in freezing. (c) Freezing to the tone: adolescent STRESS mice exhibited a significantly lower freezing response to the tone across the 3 test days compared to the $\mathrm{CON}$ mice. (d) Freezing to the CS is shown for the adult group. Freezing levels were similar in both the STRESS and the CON groups in the three tone-shock pairings. No differences were detected between the STRESS and CON groups in the contextual freezing test (e) or in the conditioned tone freezing test (f). The data were logarithmictransformed (ln-transformed). Error bars refer to $\pm \mathrm{SEM} ; n=7$ per group; $($ empty circle $)=\mathrm{CON}$; (filled square $)=$ STRESS a

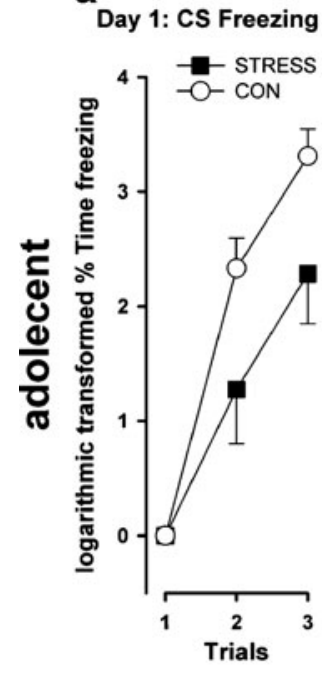

d

Day 1: CS Freezing

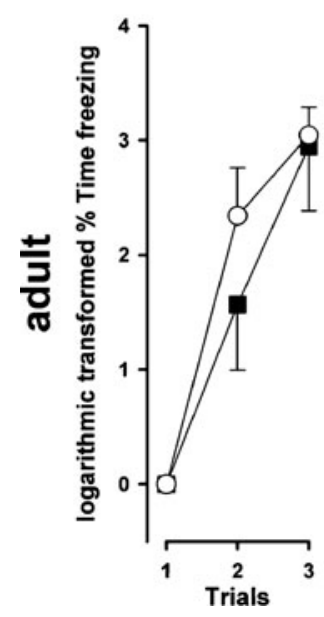

b

Day 2: Context Freezing

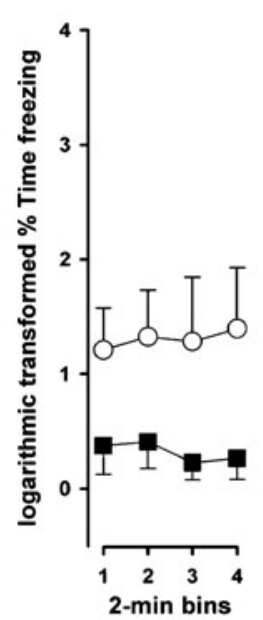

e

Day 2: Context Freezing

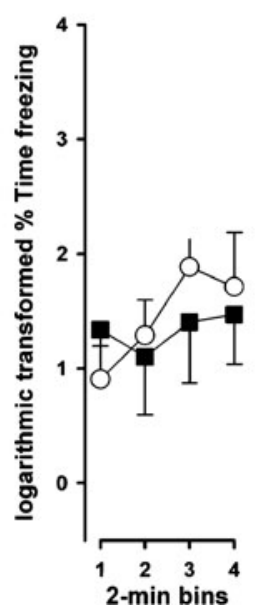

C

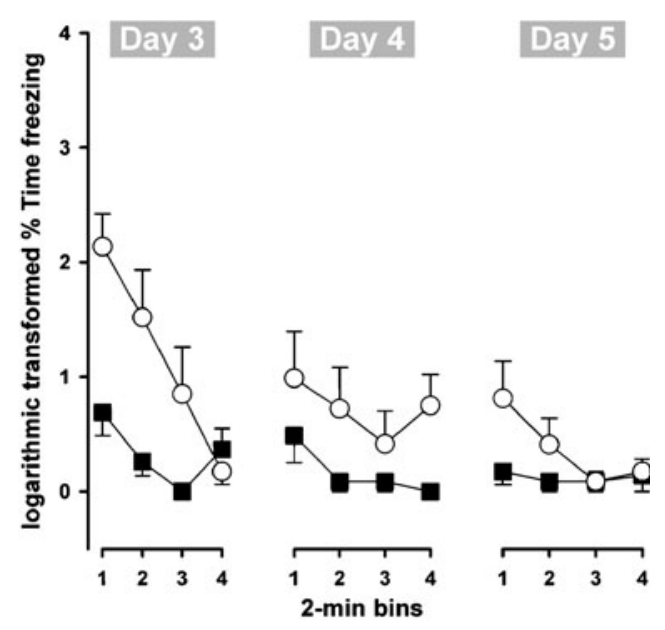

f

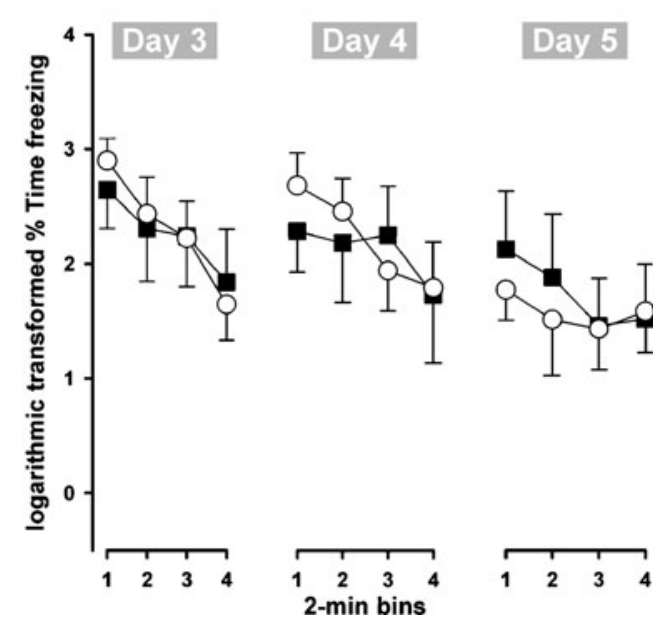

41.34; $P<0.0001$ ), indicating that avoidance learning increased in both groups as indexed by the number of avoidance responses. Moreover, the analysis revealed a significant main effect based on group $(F(1,12)=4.76 ; P<$ $0.05)$, indicating that the STRESS mice showed a reduced level of learning compared to the CON mice (Fig. 4b).

Spatial reference memory task in the water maze

\section{Adult mice}

Visible platform Separate $2 \times 4$ (group $\times$ trial) ANOVAs of latency and total distance moved in the cued test only yielded a main effect based on trial (latency, $F(3,36)=$ 12.992; $P<0.0001$; path length, $F(3,36)=8.84 ; P<0.0003)$, indicating that all of the mice learned to climb onto the escape platform, and both escape latency and distance swum decreased as a function of trials (data not shown).
Acquisition Separate $2 \times 3 \times 4$ (group $\times$ day $\times$ trial) ANOVAs of escape latency and path length yielded significant effects based on day (latency, $F(2,24)=4.62 ; P<0.03$; path length, $F(2,24)=4.73 ; P<0.02$ ) and trial (latency, $F(3,36)=4.73 ; P<$ 0.02 ; path length, $F(3,36)=3.39 ; P<0.03)$, indicating that performance improved as a function of training in both groups and that there was a general reduction in swim speed across the four test trials within each day (Fig. 5). Juvenile stress did not influence water maze performance in the adult mice, and no other main effect or interaction reached statistical significance.

Probe test A $2 \times 4$ (group $\times$ quadrant) ANOVA of the percentage of time spent per quadrant over the 60 -s probe test yielded a significant effect based on quadrant $(F(3,36)=$ 4.66; $P<0.01)$, indicating that there was a clear preference for the target quadrant (where the escape platform was located during acquisition training) in both groups. There 

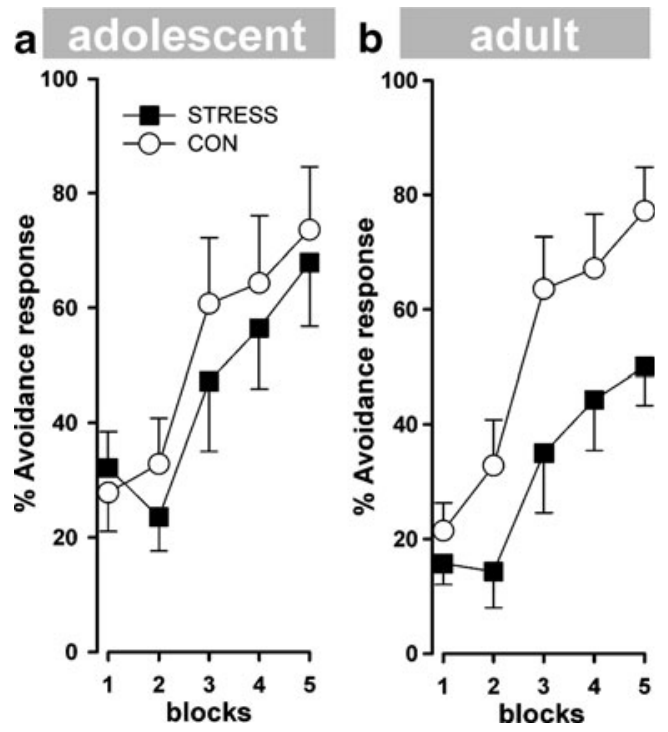

Fig. 4 Two-way active avoidance learning. Dependent measures of the number of avoidance responses are expressed in units of 20-min trial blocks. No differences in percent avoidance responses were detected between the adolescent STRESS and CON mice (a). All of the animals showed acquisition, but only the adult STRESS mice significantly differed from the CON mice in their avoidance response (b). Error bars refer to $\pm \mathrm{SEM} ; n=7$ per group; (empty circle $)=\mathrm{CON}$; (filled square $)=$ STRESS

was no apparent difference in performance between the STRESS and CON mice, and there was no statistical evidence that the search patterns among the four quadrants differed between groups [group $\times$ quadrants: $F<1$; data not shown).

Locomotor response to systemic amphetamine in the open field

\section{Adolescent mice}

The data collected in the three phases of the experiment (i.e., baseline, saline treatment, and amphetamine treatment) were analysed separately.

Baseline stage: A $2 \times 2$ ANOVA (group $\times$ bin) revealed a significant effect based on bin $(F(1,12)=78.19 ; P<$ $0.0001)$, indicating that locomotor activity decreased over time, leading to a clear habituation effect that did not differ between the STRESS and CON mice for baseline locomotor activity (Fig. 6a).

Saline stage: A $2 \times 2$ ANOVA (group $\times$ bin) revealed a significant effect based on bin $(F(1,12)=7.70 ; P<$ 0.02 ), indicating that locomotor activity decreased over time and that activity levels did not differ between the STRESS and CON mice (Fig. 6a).

Amphetamine stage: A $2 \times 8$ ANOVA (group $\times$ bin) revealed a significant effect based on bin $(F(7,84)=$
10.25; $P<0.0001)$, indicating that administration of a systemic amphetamine injection led to a general increase in locomotor activity in both groups (Fig. 6a, b). No significant effects based on group or interactions between groups and bins were observed $(F<1)$.

\section{Adult mice}

Baseline stage: A $2 \times 2$ ANOVA (group $\times$ bin) revealed a significant effect based on bin $(F(1,12)=78.19 ; P<$ 0.0001 ), indicating that locomotor activity decreased over time, reflecting a clear habituation effect that did not differ between the STRESS and CON mice for baseline locomotor activity (Fig. 6c).

Saline stage: A $2 \times 2$ ANOVA (group $\times$ bin) revealed a significant group $\times$ bin interaction $(F(1,12)=13.90 ; P<$ $0.003)$, indicating that the STRESS mice exhibited enhanced activity levels in the second 15-min bin compared to the first bin, whereas activity levels in the CON mice decreased from the first to the second bin (Fig. 6c).

Amphetamine stage: A $2 \times 8$ ANOVA (group $\times$ bin) revealed a significant effect based on bin $(F(7,84)=$ 10.56; $P<0.0001)$, indicating that administration of systemic amphetamine led to a general increase in locomotor activity in both groups (Fig. 6c). The STRESS mice, however, displayed a sensitised effect to the drug compared to the CON mice, as indicated by a significant effect based on group $(F(1,12)=11.15 ; P<$ 0.006; Fig. 6d).
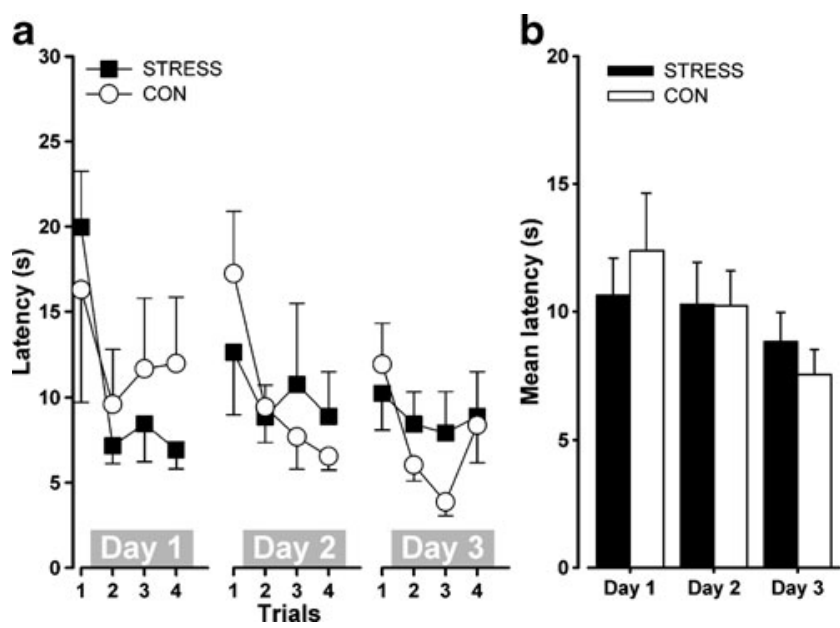

Fig. 5 Assessing reference memory in adult mice in the water maze. Mean escape latencies to reach the hidden platform across trials (in seconds) of STRESS and CON mice during 3 days of water maze acquisition training with the platform fixed in the same quadrant. The STRESS mice did not differ from the CON mice in the acquisition training of the reference memory test. Error bars refer to \pm SEM; $n=7$ per group; $($ empty circle $)=\mathrm{CON} ;($ filled square $)=$ STRESS 


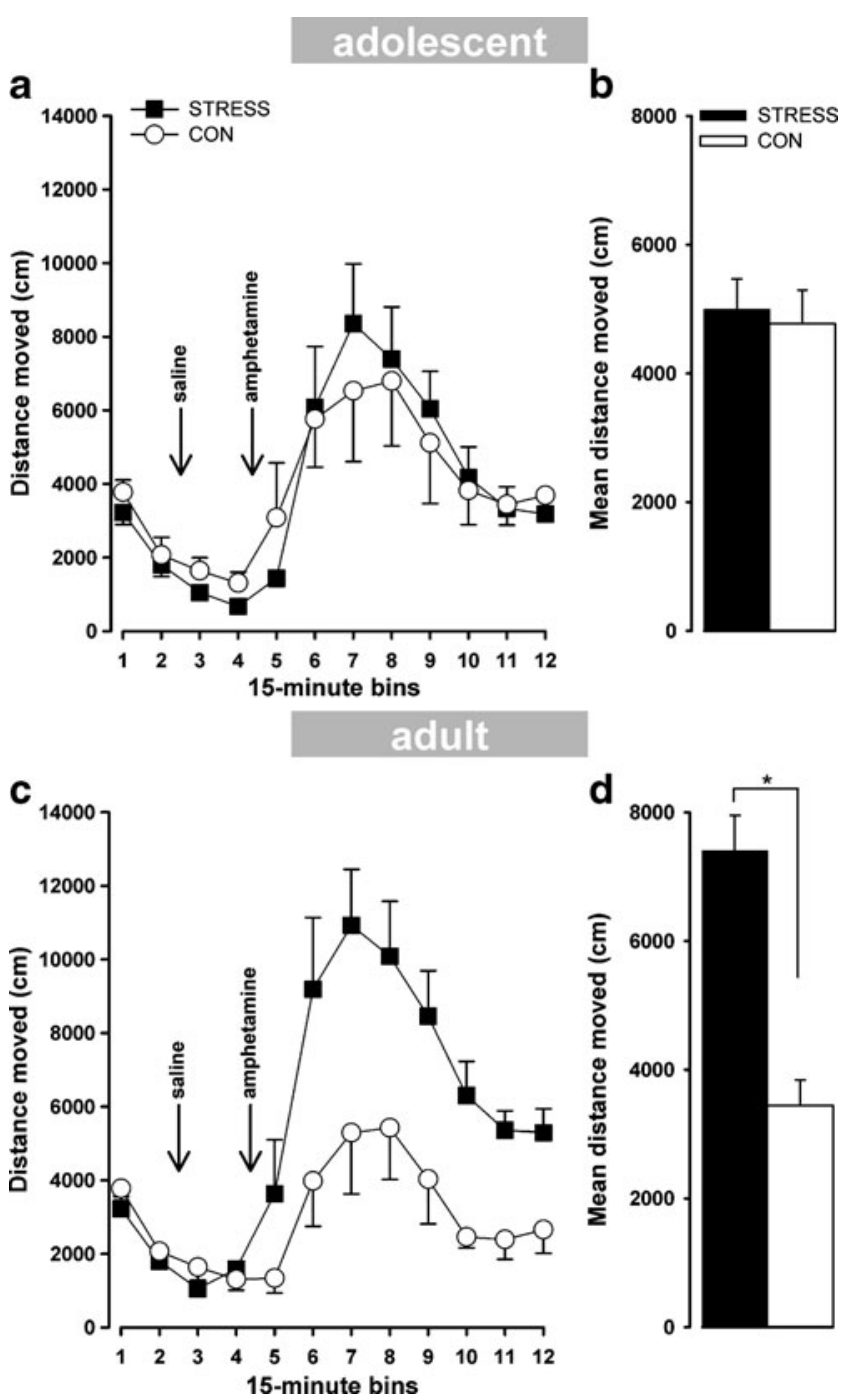

Fig. 6 Distance travelled per 15-min bin following baseline activity, saline injection, and amphetamine injection. (a) The adolescent STRESS mice did not differ from the CON mice in their locomotor activity response to baseline activity, following a saline injection or following a systemic injection of amphetamine. (c) The adult STRESS mice showed a significantly enhanced response to amphetamine compared to the $\mathrm{CON}$ mice. No differences in locomotor activity were detected during the baseline and saline stages. Insets near each graph $(\mathbf{b}, \mathbf{d})$ represent the mean distance travelled per bin collapsed across bins during the amphetamine stage to illustrate the relative augmentation of the locomotor response in the STRESS mice compared to the CON mice. ${ }^{*} P<0.05$; error bars refer to $\pm \mathrm{SEM} ; n=$ 7 per group; $($ empty circle $)=\mathrm{CON} ;($ filled square $)=\mathrm{STRESS}$

\section{Discussion}

The aim of this study was to investigate the developmental effects of juvenile stress (PND 25-29) on behaviour during two time periods: adolescence (PND 39-54) and adulthood (PND 81-138). We found a clear dissociation: adolescent mice previously exposed to juvenile stress displayed reduced anxiety-like behaviour, whereas adult mice previously exposed to juvenile stress exhibited reduced avoid- ance learning and a sensitised response to amphetamine. The data from the present study can be summarised as follows:

1. Relative to control animals, the adolescent mice that underwent juvenile stress showed low anxiety levels in the elevated plus maze, reduced freezing behaviour in a conditioned fear response paradigm and elevated locomotor activity when tested in the open field. A common theme to all of these changes might be reduced anxiety. No differences from control mice on these tests were detected when the juvenile stressed mice were tested in adulthood.

2. Juvenile stress led to impaired avoidance learning and induced marked locomotor hyperactivity in response to an amphetamine injection compared to controls only when the mice were tested during adulthood.

3. Juvenile stress had no effect on spatial learning of adult mice in the water maze.

Adolescent mice stressed during the juvenile period displayed reduced unconditioned and conditioned anxietylike behaviour in the elevated plus maze and open field and in an aversive Pavlovian fear conditioning paradigm. Interestingly, the adult mice stressed during the juvenile period did not differ from controls in these behavioural traits. One possibility to be considered regarding the more extensive effects of the stress treatment in adolescence compared with adulthood is the shorter time period between the stress procedure and the time behavioural testing commenced in the former (shorter) compared with the latter. However, the fact that the adult animals have demonstrated some effects which the adolescents have not (enhanced response to amphetamine, reduced active avoidance performance) undermines this possibility. These null effects found in the elevated plus maze, spontaneous locomotor activity tested in the open field, and freezing in the conditioning fear task in adult stressed mice are in agreement with previous reports in rats exposed to juvenile stress (Toledo-Rodriguez and Sandi 2007; Toth et al. 2008), but contrast with some previous reports demonstrating enhanced anxiety-like behaviour as shown by decreased time spent in the open arms of the elevated plus maze (Avital et al. 2006; Bazak et al. 2009; Cohen et al. 2007; Tsoory et al. 2007) and reduced locomotor activity in the open field (Avital et al. 2006; Avital and Richter-Levin 2005). The discrepant findings reported here and previously might be due to differences in the precise timing of the applied stress, the exact stress protocol, the duration of the stress procedure, the species, gender, and the exact behavioural testing procedures used. It should be noted that most of the studies performed in rats have only evaluated the effects of juvenile stress on behaviour during adulthood. 
The reduction in freezing in the conditioning fear paradigm seen in adolescent mice stressed during the juvenile period was not observed when the stressed animals were tested during adulthood. These observations are consistent with those found in adolescent and adult female rats (Toledo-Rodriguez and Sandi 2007). It should be pointed out here that it is unlikely that the freezing outcomes in adolescence are confounded by the enhanced locomotor activity seen in the open field. An analysis of the activity in the freezing boxes prior to the tone test revealed no difference between the stressed and unstressed mice (data not shown).

By directly comparing the effects of juvenile stress in the adolescent and adult stages of development, we were able to demonstrate a significant dissociation in the locomotor response to systemic amphetamine treatment. It is thus conceivable that the neural substrates involved in the locomotor reaction to psychotomimetic drugs such as amphetamine are still not fully developed and/or matured. The maturation processes that take place during the juvenile period are common in different mammalian species and occur in several brain regions that mediate age-specific neurobehavioural and physiological characteristics (Spear $2000,2004)$. The prefrontal cortex, amygdala, and hippocampus are still undergoing significant maturation during the juvenile period (Spear 2000).

Our finding that juvenile stress increased the locomotor activity response to acute amphetamine treatment $(2.5 \mathrm{mg} / \mathrm{kg})$ only when the drug was administered in adulthood, but not in adolescence, readily suggests that maturation processes are involved in the precipitation of this effect. This is consistent with previous studies in which chronic stress (PND 28-56) decreased the locomotor-activating effect of amphetamine when the exposure to amphetamine began immediately after the last day of stress (PND 57) (Kabbaj et al. 2002). Similar results were obtained in adolescent rats in response to nicotine (Mathews et al. 2008; McCormick et al. 2004, 2005). When testing began several weeks after the juvenile stress (PND 77 days), however, animals showed increased locomotor sensitisation to nicotine and increased locomotoractivating effects of amphetamine compared to controls (McCormick et al. 2004, 2005). The behavioural and neurochemical responses to various drugs of abuse differ in adolescents and adults (Adriani and Laviola 2000; Collins and Izenwasser 2004; Crews et al. 2000). Exposure to stressors in the prenatal or neonatal period has been found to increase susceptibility to the effects of many drugs in adulthood, suggesting that later drug responses may be programmed by early life events ( $\mathrm{Li}$ et al. 2003; McCormick et al. 2002; Meaney et al. 2002). However, there have been few studies examining the effects of stressors in the juvenile period and their impact on later behavioural responses to drugs at different age phases. One implication of the results of the current study is that the induction of locomotor hyperactivity after amphetamine observed in adult mice stressed during the juvenile period might indicate that stress during the juvenile period sensitises the brain to subsequent stressful situations or increases vulnerability to drug abuse. This, in turn, may be a consequence of a sensitised dopaminergic system. Moreover, animals demonstrate behavioural cross-sensitisation between stress and stimulant drugs such as amphetamine and cocaine. This effect is also known to increase activation of dopamine transmission in the medial prefrontal cortex and nucleus accumbens (Hamamura and Fibiger 1993; Kalivas and Stewart 1991; Sorg 1992).

We have shown that stress during the juvenile period impairs avoidance performance in a two-way active avoidance task in adult mice without affecting spatial reference learning in the water maze. The fact that the adult mice stressed during the juvenile period were slower in avoidance acquisition cannot necessarily be linked to emotionality since there was no effect of juvenile stress on open-field behaviour, in the elevated plus maze or in the conditioned fear paradigm. Similarly, differences in pain sensitivity are unlikely as there were no differences in the freezing paradigm to support such a possibility. In our view, the most likely explanation for the impaired avoidance response is a deficit in cognitive abilities. Having said this, one needs to bear in mind that the water maze results do not support such an interpretation. However, it is possible that the water maze is less sensitive in detecting subtle cognitive deficits. Therefore, one could speculate that the two-way active avoidance paradigm might be a more sensitive test to detect learning deficits compared to water maze acquisition. Another parsimonious explanation might be that adult mice stressed during the juvenile period have enhanced dopaminergic sensitivity, as shown by their increased behavioural sensitisation to an amphetamine injection. Thus, further studies are clearly warranted to identify the critical mechanisms underlying the distinct effects of juvenile stress on avoidance learning, which could be related to changes in dopamine transmission in critical brain circuits.

Our results thus lend some support to a similar study in rats showing that juvenile stress alters social behaviour and emotionality (Avital and Richter-Levin 2005; Tsoory et al. 2007; Tsoory and Richter-Levin 2006), but not cognitive functions (Toth et al. 2008) in adulthood.

One implication of the results of this study regards the use of this juvenile stress procedure in the context of two-hit insults in animal models of psychopathology. The two-hit hypothesis posits that an early life genetic or environmental insult sets up a neural predisposition to psychopathology, which may emerge in the presence of a subsequent insult or "second hit" later in life (Maynard et al. 2001). Such animal models involve pre- or early postnatal manipulations in the 
context of phenotyping schizophrenia and depression-like symptoms (Lipska 2004; Meyer and Feldon 2010; Weiss and Feldon 2001) and can be combined with juvenile stress as a second hit (insult). This might involve modelling, for example, the impact of stress in adolescence as a facilitator of the induction of schizophrenia symptoms. In this context, it should be noted that this mild stress protocol can lead to differing effects in adolescence and adulthood.

\section{Summary/conclusions}

The present study is the first to assess the effects of juvenile stress in mice on emotional and cognitive performance during two different time periods, adolescence and adulthood. We found a clear double dissociation. The juvenile stress protocol established here could be used as a tool to further investigate the two-hit hypothesis in the framework of animal models of psychopathologies such as schizophrenia, anxiety, and depression.

Acknowledgements The present study was supported by the Swiss Federal Institute of Technology (ETH) Zurich. The authors are also grateful to Peter Schmid for his excellent technical support and to the animal husbandry staff at the Laboratory of Behavioural Neurobiology for their maintenance of the subjects used in the experiments.

\section{References}

Adriani W, Laviola G (2000) A unique hormonal and behavioral hyporesponsivity to both forced novelty and D-amphetamine in periadolescent mice. Neuropharmacology 39:334-346

Avital A, Richter-Levin G (2005) Exposure to juvenile stress exacerbates the behavioural consequences of exposure to stress in the adult rat. Int J Neuropsychopharmacol 8:163-173

Avital A, Ram E, Maayan R, Weizman A, Richter-Levin G (2006) Effects of early-life stress on behavior and neurosteroid levels in the rat hypothalamus and entorhinal cortex. Brain Res Bull 68:419-424

Bazak N, Kozlovsky N, Kaplan Z, Matar M, Golan H, Zohar J, Richter-Levin G, Cohen H (2009) Pre-pubertal stress exposure affects adult behavioral response in association with changes in circulating corticosterone and brain-derived neurotrophic factor. Psychoneuroendocrinology 34:844-858

Cohen H, Kaplan Z, Matar MA, Loewenthal U, Zohar J, RichterLevin G (2007) Long-lasting behavioral effects of juvenile trauma in an animal model of PTSD associated with a failure of the autonomic nervous system to recover. Eur Neuropsychopharmacol 17:464-477

Collins SL, Izenwasser S (2004) Chronic nicotine differentially alters cocaine-induced locomotor activity in adolescent vs. adult male and female rats. Neuropharmacology 46:349-362

Crews FT, Braun CJ, Hoplight B, Switzer RC 3rd, Knapp DJ (2000) Binge ethanol consumption causes differential brain damage in young adolescent rats compared with adult rats. Alcohol Clin Exp Res 24:1712-1723
Hall FS (1998) Social deprivation of neonatal, adolescent, and adult rats has distinct neurochemical and behavioral consequences. Crit Rev Neurobiol 12:129-162

Hamamura T, Fibiger HC (1993) Enhanced stress-induced dopamine release in the prefrontal cortex of amphetamine-sensitized rats. Eur J Pharmacol 237:65-71

Heim C, Nemeroff CB (2001) The role of childhood trauma in the neurobiology of mood and anxiety disorders: preclinical and clinical studies. Biol Psychiatry 49:1023-1039

Heim C, Plotsky PM, Nemeroff CB (2004) Importance of studying the contributions of early adverse experience to neurobiological findings in depression. Neuropsychopharmacology 29:641-648

Isgor C, Kabbaj M, Akil H, Watson SJ (2004) Delayed effects of chronic variable stress during peripubertal-juvenile period on hippocampal morphology and on cognitive and stress axis functions in rats. Hippocampus 14:636-648

Kabbaj M, Isgor C, Watson SJ, Akil H (2002) Stress during adolescence alters behavioral sensitization to amphetamine. Neuroscience 113:395-400

Kalivas PW, Stewart J (1991) Dopamine transmission in the initiation and expression of drug- and stress-induced sensitization of motor activity. Brain Res Brain Res Rev 16:223-244

Kessler RC, Magee WJ (1993) Childhood adversities and adult depression: basic patterns of association in a US national survey. Psychol Med 23:679-690

Li Y, Robinson TE, Bhatnagar S (2003) Effects of maternal separation on behavioural sensitization produced by repeated cocaine administration in adulthood. Brain Res 960:42-47

Lipska BK (2004) Using animal models to test a neurodevelopmental hypothesis of schizophrenia. J Psychiatry Neurosci 29:282-286

Luecken LJ, Lemery KS (2004) Early caregiving and physiological stress responses. Clin Psychol Rev 24:171-191

Maslova LN, Bulygina VV, Popova NK (2002) Immediate and longlasting effects of chronic stress in the prepubertal age on the startle reflex. Physiol Behav 75:217-225

Mathews IZ, Mills RG, McCormick CM (2008) Chronic social stress in adolescence influenced both amphetamine conditioned place preference and locomotor sensitization. Dev Psychobiol 50:451459

Maynard TM, Sikich L, Lieberman JA, LaMantia AS (2001) Neural development, cell-cell signaling, and the "two-hit" hypothesis of schizophrenia. Schizophr Bull 27:457-476

McCormick CM, Kehoe P, Mallinson K, Cecchi L, Frye CA (2002) Neonatal isolation alters stress hormone and mesolimbic dopamine release in juvenile rats. Pharmacol Biochem Behav 73:7785

McCormick CM, Robarts D, Gleason E, Kelsey JE (2004) Stress during adolescence enhances locomotor sensitization to nicotine in adulthood in female, but not male, rats. Horm Behav 46:458 466

McCormick CM, Robarts D, Kopeikina K, Kelsey JE (2005) Longlasting, sex- and age-specific effects of social stressors on corticosterone responses to restraint and on locomotor responses to psychostimulants in rats. Horm Behav 48:64-74

Meaney MJ, Brake W, Gratton A (2002) Environmental regulation of the development of mesolimbic dopamine systems: a neurobiological mechanism for vulnerability to drug abuse? Psychoneuroendocrinology 27:127-138

Meyer U, Feldon J (2010) Epidemiology-driven neurodevelopmental animal models of schizophrenia. Prog Neurobiol 90:285-326

Penza KM, Heim C, Nemeroff CB (2003) Neurobiological effects of childhood abuse: implications for the pathophysiology of depression and anxiety. Arch Womens Ment Health 6:15-22

Pohl J, Olmstead MC, Wynne-Edwards KE, Harkness K, Menard JL (2007) Repeated exposure to stress across the childhoodadolescent period alters rats' anxiety- and depression-like 
behaviors in adulthood: the importance of stressor type and gender. Behav Neurosci 121:462-474

Prut L, Belzung C (2003) The open field as a paradigm to measure the effects of drugs on anxiety-like behaviors: a review. Eur J Pharmacol 463:3-33

Richmond MA, Murphy CA, Pouzet B, Schmid P, Rawlins JN, Feldon J (1998) A computer controlled analysis of freezing behaviour. J Neurosci Meth 86:91-99

Sorg BA (1992) Mesocorticolimbic dopamine systems: crosssensitization between stress and cocaine. Ann NY Acad Sci 654:136-144

Spear LP (2000) The adolescent brain and age-related behavioral manifestations. Neurosci Biobehav Rev 24:417-463

Spear LP (2004) Adolescent brain development and animal models. Ann NY Acad Sci 1021:23-26

Tirelli E, Laviola G, Adriani W (2003) Ontogenesis of behavioral sensitization and conditioned place preference induced by psychostimulants in laboratory rodents. Neurosci Biobehav Rev 27:163-178

Toledo-Rodriguez M, Sandi C (2007) Stress before puberty exerts a sex- and age-related impact on auditory and contextual fear conditioning in the rat. Neural Plast 2007:71203
Toth E, Avital A, Leshem M, Richter-Levin G, Braun K (2008) Neonatal and juvenile stress induces changes in adult social behavior without affecting cognitive function. Behav Brain Res 190:135-139

Tsoory M, Richter-Levin G (2006) Learning under stress in the adult rat is differentially affected by 'juvenile' or 'adolescent' stress. Int J Neuropsychopharmacol 9:713-728

Tsoory M, Cohen H, Richter-Levin G (2007) Juvenile stress induces a predisposition to either anxiety or depressive-like symptoms following stress in adulthood. Eur Neuropsychopharmacol 17:245-256

Weiss IC, Feldon J (2001) Environmental animal models for sensorimotor gating deficiencies in schizophrenia: a review. Psychopharmacology (Berl) 156:305-326

Weiss EL, Longhurst JG, Mazure CM (1999) Childhood sexual abuse as a risk factor for depression in women: psychosocial and neurobiological correlates. Am J Psychiatry 156:816-828

Yee BK, Hauser J, Dolgov VV, Keist R, Mohler H, Rudolph U, Feldon J (2004) GABA receptors containing the alpha5 subunit mediate the trace effect in aversive and appetitive conditioning and extinction of conditioned fear. Eur J Neurosci 20:1928-1936 